Association for Information Systems

AIS Electronic Library (AISeL)

Wirtschaftsinformatik Proceedings 2003

Wirtschaftsinformatik

September 2003

\title{
Strukturierung von Lerninhalten mit dem Ziel ihrer Wiederverwendung: ist der Spagat zwischen Didaktik und Informationstechnik zu bewältigen?
}

Berit Jungmann

Technische Universität Dresden, berit.jungmann@wiim.wiwi-tu-dresden.de

Eric Schoop

Technische Universität Dresden

Karin Wirth

Universität Leipzig

Fritz Klauser

Universität Leipzig

Follow this and additional works at: http://aisel.aisnet.org/wi2003

\section{Recommended Citation}

Jungmann, Berit; Schoop, Eric; Wirth, Karin; and Klauser, Fritz, "Strukturierung von Lerninhalten mit dem Ziel ihrer Wiederverwendung: ist der Spagat zwischen Didaktik und Informationstechnik zu bewältigen?" (2003). Wirtschaftsinformatik Proceedings 2003. 34.

http://aisel.aisnet.org/wi2003/34

This material is brought to you by the Wirtschaftsinformatik at AIS Electronic Library (AISeL). It has been accepted for inclusion in Wirtschaftsinformatik Proceedings 2003 by an authorized administrator of AIS Electronic Library (AISeL). For more information, please contact elibrary@aisnet.org. 
In: Uhr, Wolfgang, Esswein, Werner \& Schoop, Eric (Hg.) 2003. Wirtschaftsinformatik 2003: Medien - Märkte - Mobilität, 2 Bde. Heidelberg: Physica-Verlag

ISBN: 3-7908-0111-9 (Band 1)

ISBN: 3-7908-0116-X (Band 2)

(C) Physica-Verlag Heidelberg 2003 


\title{
Strukturierung von Lerninhalten mit dem Ziel ihrer Wiederverwendung: ist der Spagat zwischen Didaktik und Informationstechnik zu bewältigen?
}

\author{
Berit Jungmann, Eric Schoop \\ Technische Universität Dresden \\ Karin Wirth, Fritz Klauser \\ Universität Leipzig
}

Zusammenfassung: Die Potenziale von XML (Mächtigkeit und Flexibilität der semantischen Modellierung sowie die Möglichkeit zur Wiederverwendung ausgezeichneter Komponenten) werden seit einiger Zeit auch im Kontext von ELearning diskutiert. Nahezu unberücksichtigt bleibt die didaktische Dimension: Pädagogen werden in der Regel erst bei der Erstellung der Contents hinzugezogen. Die Autoren zeigen, dass sich der Einsatz von XML im Kontext von ELearning von anderen Anwendungsgebieten qualitativ dadurch unterscheidet, dass Lerninhalte eine didaktische Funktion erfüllen und daher strukturiert aufbereitet werden müssen. Ein darauf aufsetzendes XML-basiertes Content Management kann grundsätzlich die Wiederverwendung von Lerninhalten ermöglichen und damit neben der didaktischen Effizienz auch die ökonomische Dimension neu in das Zentrum der Diskussion rücken, setzt jedoch weitergehende Modellierungskonzepte voraus.

Schlüsselworte: XML, Didaktik, E-Learning, Lerninhalt, Wiederverwendung

\section{Wiederverwendung von Lerninhalten - eine pädagogische und technische Herausforderung}

Soll E-Learning über das Angebot „Elektronischer Bücher“ hinausgehen, so ist unter anderem eine multimediale Aufbereitung notwendig. Im Gegensatz zu traditionellen Medien ist jedoch mit einem bis zu zehnfachen Zeitaufwand für die Entwicklung von multimedialen Lernmaterialien zu rechnen. Die Entwicklungskosten für eine E-Learning-Stunde werden zur Zeit mit 2.000 bis 20.000 Euro veranschlagt [Häf ${ }^{+}$2] . Angesichts dessen kann es nicht verwundern, dass versucht 
wird, Inhalte modular zu gestalten, damit sie später mehrfach verwendet - wiederverwendet - werden können, um so verbesserte Skalenerträge zu erreichen.

In der Softwareentwicklung spielt das Thema Wiederverwendung bereits seit ca. 50 Jahren eine entscheidende Rolle. Unter dem Begriff der „SoftwareWiederverwendung“ wird dabei ,das erneute Nutzen existierender Artefakte und angesammelten Wissens bei der Entwicklung neuer Anwendungssysteme" verstanden mit dem Ziel, den Erstellungs- und Pflegeaufwand zu reduzieren [Griff98, S. 16; Reza95, S. 221f.; Diet02, S. 7].

Ziele der Wiederverwendung sind folglich die

- Erhöhung der Produktivität,

- Verbesserung der Produktqualität,

- Verkürzung der Entwicklungszeit und

- Reduktion der Kosten.

Im Zentrum stehen drei Gestaltungsfaktoren für Wiederverwendung: erstens die rein technische Konzeption, zweitens die Berücksichtigung der organisatorischen Ausgestaltung und drittens die Sicherstellung der Wiederverwendung von Komponenten durch das Management. Weit verbreitet ist die Ansicht, dass die Wiederverwendung heute kein technisches, sondern vielmehr ein Organisations- und Managementproblem darstellt [Balz98, S. 650].

Im Bereich des E-Learning können folgende Formen der Wiederverwendung unterschieden werden [in Anlehnung an KlSt01]:

- Wiederverwendung der selben Inhalte für unterschiedliche Benutzergruppen (z. B. Studierende, Schüler, Arbeitnehmer),

- Wiederverwendung der selben Inhalte für unterschiedliche Lehr- und Lernformen (z. B. Selbststudium, Präsenzveranstaltung),

- Wiederverwendung der selben Inhalte für unterschiedliche Medien (z. B. Internet, CD-ROM, Print) und

- Wiederverwendung von ,eigenen“ und ,externen“ Inhalten (d. h. von anderen Autoren erstellte Inhalte).

Aus technischer Perspektive bezieht sich die Wiederverwendbarkeit auf den verwalteten „Content“, der allzu häufig fälschlicherweise mit dem „Lerninhalt“ gleichgesetzt wird ${ }^{1}$. Diese Begriffe sind deshalb differenziert zu betrachten und zu verwenden.

\footnotetext{
Streng definitorisch genommen sind beide Begriffe zudem von dem „,fachwissenschaftlichen Stoff", dem Inhalt der zu Grunde liegenden Bezugsdisziplin, zu unterscheiden [ $\left.\operatorname{Jun}^{+} 02\right]$.
} 
Content stellt aus informationstechnischer Sicht Inhaltselemente jeglicher Art dar, wie z. B. strukturierte Daten oder formatierte Dokumente, Grafiken sowie Animationen [RoRi01]. In struktureller Hinsicht werden zwei Ausprägungen von Content unterschieden: Einerseits existieren sogenannte atomare Grundbausteine, die nicht in weitere Bestandteile zerlegt werden können. Auf der anderen Seite gibt es Komponenten, die aus verschiedenen atomaren Objekten zusammengesetzt sind. Informationstechnisch kann Content sowohl atomar im Sinne eines einzelnen Lernobjekts als auch zusammengesetzt im Sinne einer Kombination von Lernobjekten gestaltet sein.

Lerninhalt hingegen ist stets didaktisch aufbereiteter Content und verweist damit auch auf ganz bestimmte Methoden, Medien und vor allem auf zu Grunde liegende Lernziele und für die Zielrealisierung notwendige Lernprozesse. Dabei ist zu berücksichtigen, dass die Inhalte, Methoden, Medien und Lernprozesse nicht in beliebiger Weise miteinander kombiniert werden können, sondern in einer wechselseitigen Abhängigkeit stehen und didaktisch aufeinander abgestimmt werden. Bereits im Stadium der Konstruktion bzw. Planung von Lernangeboten werden Entscheidungen über Auswahl, Anordnung und Umsetzung des fachwissenschaftlichen Inhalts getroffen, die den späteren Einsatz innerhalb einer Lernsituation beeinflussen [Acht01; $\mathrm{Kla}^{+} 02$; Klau02]. Eine beabsichtigte Wiederverwendung muss also bereits in der Planungsphase berücksichtigt werden.

Ausgangsbasis unseres Ansatzes ist die Feststellung, dass die Wiederverwendung von Lerninhalten von der didaktischen Intention abhängig ist. Bei der Betrachtung dessen, was von wem mit welchem Ziel wiederverwendet wird, fällt auf, dass die von Balzert definierte Voraussetzung, dass Technik, Organisation und Management zusammenwirken [Balz92, S. 639] um die Didaktik erweitert werden muss (vgl. Abbildung 1).

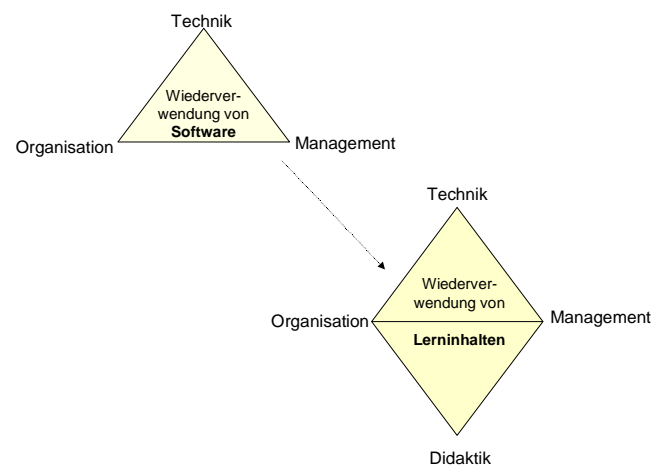

Abbildung 1: Anforderungen an die Wiederverwendung von Lerninhalten

Die Wiederverwendung von Lerninhalten muss als Herausforderung angesehen werden, die eine enge interdisziplinäre Zusammenarbeit zwischen Informations- 
management und Didaktik verlangt. Im Folgenden zeigen wir wesentliche Aspekte einer interdisziplinären Sichtweisen auf und schlagen einen gemeinsamen Lösungsweg zur Strukturierung und Wiederverwendung von Lerninhalten vor.

Aus informationstechnischer Sicht bildet XML (eXtensible Markup Language) dabei die Grundlage für die Strukturierung von Lerninhalten (siehe Kapitel 2), die zu Grunde liegenden didaktischen Ansätze werden in Kapitel 3 vorgestellt. Im vierten Kapitel werden didaktische und informationstechnische Konzepte zum Lösungsansatz für die Ausgestaltung wiederverwendbarer Lerninhalte zusammengeführt.

\section{Informationstechnische Basis: Strukturdefinitionen für XML}

Kennzeichnend für XML ist die Trennung von Struktur, Inhalt und Layout. Die Präsentation der Inhalte erfolgt losgelöst von ihrer Erstellung durch ein nachträglich zugeordnetes Stylesheet, das anhand der Strukturinformationen automatisch ein Layout zuweist.

XML wird im E-Learning insbesondere in folgenden Anwendungsbereichen eingesetzt [Luc $\left.{ }^{+} 02\right]$ :

- Abbildung von Metadaten,

- Interoperabilität von Lernsystemen (z. B. Learning Management Systeme (LMS), Autorensysteme),

- Austausch von Benutzerdaten und

- Strukturierung des Lerninhalts.

Aktuelle Bestrebungen, Kriterien für die Wiederverwendung zu entwickeln, konzentrieren sich primär auf den Bereich der Metadaten [vgl. dazu die Übersicht in Luc $^{+} 02 ;$ PaAd01]. Die Struktur und die Semantik der Dokumente werden dagegen kaum berücksichtigt.

Die Struktur kann durch Document Type Definitions (DTDs) oder XML Schema ${ }^{2}$ festgelegt werden, die als eine Art Vorlage zur Erstellung neuer Dokumente dienen [GoPr00]. Nicht weiter strukturierbare Informationen (wie z. B. Audio) werden mit Metadaten versehen, um einen schnellen und präzisen Zugriff auf deren Inhalt zu gewährleisten.

2 Im weiteren Verlauf des Beitrags wird das DTD-Konzept zu Grunde gelegt. 
Der wesentliche Vorteil der DTD-konformen Dokumentauszeichnung ist neben der „vorlagenorientierten“ Inhaltsstrukturierung die Möglichkeit, die ausgezeichneten Inhalte automatisch identifizieren und weiterverarbeiten zu können (detailliertes Klassifizieren, Suchen, Kombinieren). Eine Strukturierung von Dokumenten ist technisch gesehen jedoch nur in einer Tiefe sinnvoll, in der die Strukturinformationen auch wirklich weiterverarbeitet werden.

In struktureller Hinsicht können DTDs verschiedene Ausprägungen zwischen Liberalität und Restriktivität annehmen [Lobi00]. Dargestellt ist dieses Kontinuum in Abbildung 2. Im Folgenden werden die Ausprägungen beispielhaft umrissen.

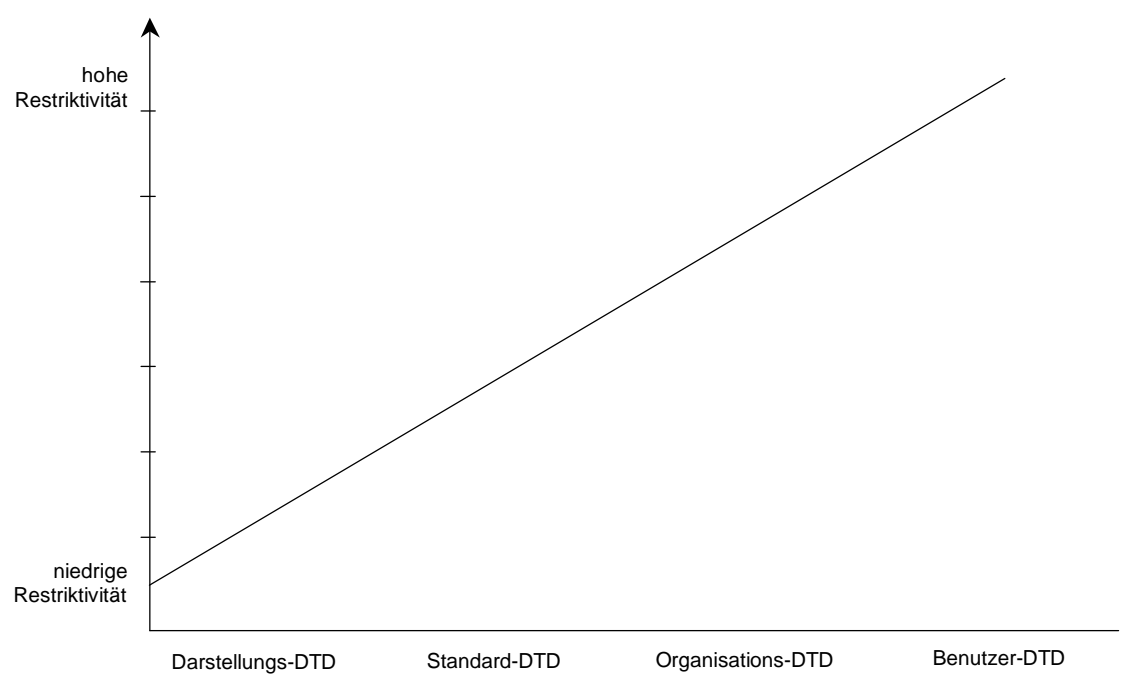

Abbildung 2: Verhältnis von Liberalität und Restriktivität bei DTDs [Lobi00]

Ein Beispiel für eine darstellungsbezogene DTD ist HTML (Hypertext Markup Language). Strukturelle oder semantische Aspekte werden hier nicht berücksichtigt - eine Spezifikation der Struktur erfolgt lediglich anhand der Darstellung.

HTML ist damit ungeeignet für:

- den Austausch von Komponenten,

- die Wiederverwendung von Komponenten,

- die automatisierte Verarbeitung und

- die Auswertung des Inhalts der Dokumente.

HTML wird für WBT-Lernsoftware seit längerer Zeit genutzt, ist aber für größere Projekte ungeeignet. 
Standardisierte DTDs dienen insbesondere dem elektronischen Austausch von Daten. Hinsichtlich der Struktur wird versucht, den „kleinsten gemeinsamen Nenner aller potentiellen proprietären DTDs innerhalb einer Domäne“ [Lobi00] abzubilden. Kennzeichnend ist der hohe Grad an Liberalität. Bekannte Standard-DTDs sind u. a. ISO 12083 (für wissenschaftliche Publikationen) und TEI (für geisteswissenschaftliche Texte), branchenspezifische Anwendungen sind z. B. SCORM (im Bereich E-Learning) [ADL03].

An einen Standard werden die folgenden allgemeinen Anforderungen gestellt [Fran00; Krcm00]:

- generelle Gültigkeit,

- Angemessenheit und Vollständigkeit,

- Stabilität,

- Flexibilität,

- einfache Handhabung und

- Verfügbarkeit von Werkzeugen.

Eine wesentliche Grundlage für die Akzeptanz eines Standards stellt die generelle Gültigkeit dar. Individuelle Anforderungen müssen weitestgehend abgedeckt werden. Ein dem Verwendungszweck gerecht werdender Detaillierungs- und Formalisierungsgrad sowie die Berücksichtigung aller sich aus dem Verwendungszweck ergebender Anforderungen sind weitere, nicht zu unterschätzende Forderungen. Veränderliche Bedingungen werden bei der Entwicklung von neuen Standards vorausschauend berücksichtigt, um für einen langen Zeitraum gültige Aussagen festzulegen. Weiterhin muss die Voraussetzung geschaffen werden, die Spezifikationen eines Standards flexibel an neue Anforderungen anzupassen. Einfache Handhabung und Verfügbarkeit von Werkzeugen stellen daher notwendige Rahmenbedingungen dar.

Organisations-DTDs strukturieren Daten für organisationsinterne Verarbeitungen, werden jedoch nicht für die direkte Datenproduktion genutzt. Sie sind stärker restringiert als Standard-DTDs, dennoch weniger spezifisch als Benutzer-DTDs. Sie werden in diesem Beitrag nicht weiter berücksichtigt.

Durch Benutzer-DTDs wird die Konsistenz neu entwickelter Daten gewährleistet. Für die Beschreibung und Strukturierung von Lerninhalten eignen sich diese in besonderem Maße, da sie eine Gestaltung unter spezifischer Zielstellung möglich machen. Allerdings ist dabei auf pädagogisch fundierte Ansätze der Strukturierung und Sequenzierung von Lerninhalten zurückzugreifen. Damit kann die didaktische Struktur eines Lernangebots in die informationstechnische Struktur, die DTD, umgesetzt werden (die Berücksichtigung semantischer Aspekte wird in Kapitel 4 
am Beispiel unserer im Projekt IMPULS ${ }^{\mathrm{EC}} 3$ entwickelten problemorientierten PBL-DTD vorgestellt). Für eine Wiederverwendung von Lerninhalten ist die DTD zusätzlich mit Metadaten semantisch anzureichern. Benutzer-DTDs sind in der Lage, trotz der spezifischen Ausrichtung gängige Standards (z. B. SCORM) zu berücksichtigen.

Geeignete, an die Anwendung adaptierte XML-Editoren unterstützen die Qualitätssicherung. Es erfolgt eine Überprüfung im Hinblick auf die Vollzähligkeit der curricularen und didaktischen Elemente, Komponenten und Funktionen. Die Erstellung der Inhalte wird vom Fachautor in der durch die DTD vorgegebenen Struktur vorgenommen. Pflichtelemente wie beispielsweise angestrebte Lernziele oder erwartetes bzw. benötigtes Vorwissen müssen aus diesem Grund vom Autor angegeben werden, ansonsten sind die Dokumente nicht valide. Für die didaktische Aufbereitung dieser Struktur stellen wir die folgenden Ansätze vor.

\section{Didaktische Ansätze zur Sequenzierung von Lerninhalten}

Ein elaborierter und empirisch umfangreich überprüfter Ansatz zur Sequenzierung von Lerninhalten ist Problem-Based Learning. Die Grundlagen des ProblemBased Learning wurden in den 60-er Jahren entwickelt und wurden seitdem weltweit rezipiert, angewandt sowie für den pädagogisch fundierten Umgang mit modernen Informations- und Kommunikationsmedien weiterentwickelt [vgl. Klau98b; für E-Learning insbesondere Klau02]. Problem-Based Learning zählt zu den situierten Ansätzen, für die folgende Gemeinsamkeiten charakteristisch sind.

Unter dem Begriff „Situierte Ansätze“ werden in der Literatur jene Konzepte zusammengefasst, die eine gemeinsame Grundauffassung über Lehr-Lern-Prozesse vertreten, insbesondere im Hinblick auf die Bedeutung der Umgebungsbedingungen. Lernen findet danach grundsätzlich in einem Kontext statt, dessen soziale, gegenständliche, motivationale und emotionale Faktoren die Art und Weise, wie gelernt wird, sowie die Anwendbarkeit des Wissens entscheidend mitbestimmen [Klau98a, S. 4]. Daraus entsteht die Forderung nach Authentizität ei-

3 Das Projekt IMPULS ${ }^{\mathrm{EC}}$ (Interdisziplinäres multimediales Programm für universitäre Lehre und selbstorganisiertes Lernen: Electronic Commerce) wird vom BMBF im Rahmen des Programms „Neue Medien in der Bildung“ gefördert. Jede Partneruniversität übernimmt spezifische Rollen im Entwicklungsprozess. Die hier vertretenen Lehrstühle haben folgende Rollen inne: Lehrstuhl für Berufs- und Wirtschaftspädagogik, Universität Leipzig: didaktisch-methodische und curriculare Begleitung, Evaluation; Lehrstuhl für Wirtschaftsinformatik, insbesondere Informationsmanagement, Technische Universität Dresden: DTD-Engineering, Content Management und Aufbau der Lernplattform. 
ner Lernumgebung, d. h. die Lernsituationen sind den Anwendungssituationen möglichst ähnlich zu gestalten [Man ${ }^{+} 02$, S. 141].

Situierte Ansätze zeichnen sich zudem dadurch aus, dass die Gestaltung von Lernumgebungen am beabsichtigten Lernprozess orientiert wird. Diese Herangehensweise stützt sich auf veränderte Annahmen zum Lernprozess [vgl. Klau98c, S. 333]. Danach sind Lernumgebungen, die auf die bloße Übermittlung von Wissen gerichtet sind und den Lernenden zum passiven Konsumenten degradieren, kaum effektiv. Lernen wird stattdessen als aktive und konstruktive Auseinandersetzung des Lernenden mit dem Lernangebot betrachtet. Lernende konstruieren ihr Wissen, indem sie wahrnehmungsbedingte Erfahrungen interpretieren und sich mit dargebotenen Informationen, Problemen und Situationen zielbezogen auseinandersetzen. Dazu bedarf es einer zielgerichteten Steuerung der (Lern-)Aktivitäten durch den Lernenden. Die zentrale Funktion eines Lernangebots besteht darin, Lernaktivitäten zu initiieren, die Lernenden zur aktiven Auseinandersetzung mit dem Lernangebot zu motivieren sowie beabsichtigte Lernprozesse zu fördern und angemessen zu unterstützen. Effektive Lehr-Lern-Prozesse vollziehen sich in sozialer Interaktion und Kommunikation ebenso wie in der Auseinandersetzung mit historischen und kulturellen Hintergründen der zu Grunde liegenden Informationen, Probleme und Situationen [ $\mathrm{Man}^{+} 02$, S. 140].

An das Lernen und die Ergebnisse von Lehr-Lern-Prozessen werden veränderte Anforderungen gestellt. Die neuen Medien, insbesondere der Computer und das Internet bieten die technischen Voraussetzungen, um die Stellung des Lernens in der Gesellschaft zu verändern. Ansätze situierten Lernens betonen den hohen Stellenwert der Neuen Medien für den Lernprozess [vgl. für Problem-Based Learning Klau02, S. 4; für die situierten Ansätze allgemein $\mathrm{Man}^{+} 02$; mit Bezug auf DuJo92].

Situierte Ansätze betonen zugleich die Bedeutung des Problemlösens [Man ${ }^{+} 02$, S. 143]. Beim Problem-Based Learning wird Lernen als generatives Problemlösen geplant und initiiert. Der Lernende wird zu Beginn der Lernsequenz mit einer komplexen Problemstellung konfrontiert und generiert in der Folge sein Wissen im Prozess der Problembearbeitung. Indem er die Problemstellung bearbeitet, identifiziert und lokalisiert der Lernende die benötigten Informationen, macht sie sich systematisch verfügbar und bewertet sie im Hinblick auf die Möglichkeit, einen Beitrag zur Lösung des Problems bereitzustellen. Der Lernende sammelt nicht ziellos Informationen, sondern nutzt sie zur Suche und zur Präsentation einer Problemlösung.

Die komplexe Problemstellungen stehen als curricularer und didaktischmethodischer Bezugs- und Ausgangspunkt am Beginn einer Lernsequenz. Sie sind vorzugsweise realitätsnah und authentisch zu gestalten und in einen subjektiv bedeutsamen Kontext einzubetten. Dabei werden die Kriterien zur Gestaltung von Curricula mit denen der Lehr-Lern-Prozessgestaltung verknüpft (Verknüpfung von Mikro- und Makrosequenzierung). Diese Verknüpfung bezieht sich nicht nur 
auf eine fachspezifische, d. h. ziel- und inhaltsbezogene Dimension, sondern erstreckt sich darüber hinaus auf institutionelle Bedingungen [vgl. Klau98b, S. 334].

Wird von der Annahme ausgegangen, dass Lernen ein aktiver, konstruktiver Prozess ist, der in einer Lernumgebung und innerhalb einer Lernsituation stattfindet, so können die didaktischen Funktionen des Lernangebots folgendermaßen eingeteilt werden:

Initiierung des Lernprozesses. Der Lernende erhält ein komplexes Problem mit einem Arbeitsauftrag in einem authentischen Kontext. Diese komplexe Problemstellung dient als Ausgangspunkt für den Lernenden, die Informationen aktiv in seine kognitive Struktur zu integrieren bzw. die kognitive Struktur um diese Informationen zu erweitern.

Diese didaktische Funktion korrespondiert mit der Phase der Orientierung im Lernprozess, in der der Lernende eine Situationsbestimmung vornimmt, individuelle Ziele entsprechend seiner Interessen formuliert sowie eine erste, vorläufige Planung seiner Lernaktivitäten durchführt.

Bereitstellung der Inhalte. Die benötigten Informationen werden dem Lernenden vom Lernangebot zur Verfügung gestellt. Diese didaktisch aufbereiteten Inhalte nutzt der Lernende, um sein Wissen zielorientiert anhand der Problemstellung aktiv zu konstruieren.

Innerhalb dieser Phase identifiziert und bewertet der Lernende die verfügbaren Informationen im Hinblick auf seine formulierten Ziele, nutzt und verteilt die ihm zur Verfügung stehenden Ressourcen, systematisiert und klassifiziert die erworbene Information, realisiert seine Strategien zur Bearbeitung der Problemstellung und kontrolliert bzw. korrigiert sie entsprechend dem wahrgenommenen Lernfortschritt.

Kontrolle des Lernerfolgs. Aufgaben zur Kontrolle des Lernerfolgs haben innerhalb einer Lernumgebung eine zweifache Funktion. Zunächst kann der Lernende während der Bearbeitung der Aufgaben und beim Vergleich seiner individuellen Lösung mit einer Musterlösung oder verschiedenen Ergebnisalternativen seine Defizite und Schwächen, aber auch seinen individuellen Lernfortschritt diagnostizieren. Anhand von Aufgaben kann er eine Kontrolle und Bewertung seiner eigenen Lernaktivitäten vornehmen. Diese Selbstüberprüfung wird innerhalb der konkreten Lernsituation von einem individuellen, fachlich geprägten Feedback begleitet, das neben der korrekten Lösung auch den Prozess der Lösungserstellung sowie mögliche Fehler bzw. Fehlvorstellungen umfasst. Zum anderen dient die Lernerfolgskontrolle zur Erfassung und Bewertung der Lernerleistung und damit zur Selektion bzw. Qualifikation. Entsprechende Aufgaben müssen fachlich einem repräsentativen Ausschnitt des zu Grunde liegenden Lerninhalts entsprechen und organisationstechnisch verwaltbar sein. Neben der richtigen Lösung muss die relative Stellung innerhalb der gesamten Kontrolle angegeben werden (können). 
Für die Strukturierung und Sequenzierung von Lerninhalten können die Anforderungen der Ansätze situierten Lernens folgendermaßen zusammen gefasst werden: Multimedial umgesetzte, internetbasierte Lernangebote sind so aufzubereiten, dass sie die Phasen des individuellen Lernprozesses angemessen unterstützen. In jeder Phase des Lernprozesses besitzt ein Lernangebot eine spezielle Funktion. Diese Funktion gilt es bei der Strukturierung und Sequenzierung von Lerninhalten zu berücksichtigen. XML bietet die technische Möglichkeit, Lernangebote zu erstellen, deren einzelne Komponenten didaktische Funktionen abbilden, die automatisiert auswertbar ausgezeichnet werden können.

\section{Synergien von informationstechnischen und didaktischen Konzepten}

\subsection{Nutzung von XML zur Strukturierung von Lerninhalten}

XML bietet die Möglichkeit der anforderungsgerechten Strukturierung, durch die eine geeignete Wiederverwendung realisierbar ist. Dazu müssen jedoch didaktische Prinzipien und Strukturen festgelegt und in Modellen abgebildet werden. Bei dieser semantischen Modellierung werden eine inhaltsorientierte und eine strukturorientierte Sequenzierungshierarchie unterschieden [Jung03; Schr97].

Während die strukturorientierte Hierarchie anwendungsunabhängig ist (typische Elemente wären z. B. Kapitel mit Absätzen), wird die inhaltsorientierte Hierarchie konkret auf die spezifische Anwendung ausgerichtet. Typspezifische semantische Komponenten werden als strukturgebende Elemente in der Dokumenthierarchie verankert. Vorteile dieses Ansatzes sind [Schr97]:

- präzisere Dokumenttypisierung auf Strukturebene und

- einfacherer Zugriff auf inhaltliche Bestandteile.

Ein Beispiel für eine strukturorientierte Hierarchie ist die SCORM-DTD der ADLInitiative [ADL03]. Sie dient der Beschreibung austauschbarer Lernobjekte und vereinigt Lösungen verschiedener anderer Standards, jedoch auf einer sehr hohen abstrakten Ebene. Eine Standardisierung sollte jedoch auch ,unterhalb“ der Standardisierungsschwelle (beispielsweise unternehmens- bzw. projektintern) vorgenommen werden. Unser didaktisch akzentuiertes Strukturmodell stellt einen Lösungsansatz auf dieser Ebene dar und zeichnet sich durch eine inhaltsorientierte Hierarchie aus.

Alternative Vorschläge zur Strukturierung von Lerninhalten mit DTDs sind veröffentlicht als: 
- Educational Markup Language (EML),

- Learning Material Markup Language (LMML) und

- TeachML.

Dort werden im Gegensatz zu unserem Vorschlag die Ansätze des Problem-Based Learning nicht genügend berücksichtigt. Diese dem Beitrag zu Grunde gelegte $P B L$-DTD (Problem Based Learning-DTD) liegt mittlerweile in einer bereits im Einsatz erprobten Version als Ergebnis des BMBF-Förderprojektes IMPULS ${ }^{\mathrm{EC}}$ vor [konkret zur DTD vgl. Jun $\left.{ }^{+} 03\right]$.

Bei einer didaktisch akzentuierten Entwicklung von Lernangeboten mit XML ergeben sich für die Gestaltung der DTDs folgende Fragestellungen:

- Welche medialen Präsentationsmöglichkeiten für Lernmaterialien sind für didaktisch-methodische Komponenten möglich und welche davon sind den Autoren der Lernangebote jeweils als Umsetzungsmöglichkeit anzubieten?

- Welche Phasen im Lernprozess gilt es mit dem Lernangebot zu unterstützen? Welche Funktion nimmt die jeweilige Lernsequenz innerhalb dieser Phasen ein? Welche Struktur bzw. welcher Aufbau liegt allen Lernangeboten zu Grunde? Welche didaktisch-methodischen Komponenten sind innerhalb dieser Struktur zur Realisierung eines lernerangemessenen Lernangebots erforderlich?

- Welche Größe bzw. Granularität ist für Lernangebote im Hinblick auf Qualifizierungs- und Zertifizierungsmöglichkeiten, fachlich komplexe Lernsequenzen sowie beabsichtigte Lernaktivitäten und erwartete Lernerkonzentration didaktisch sinnvoll?

Die Suche nach der optimalen Größe (Granularität) von Lerneinheiten ist bei der Entwicklung computerunterstützter Lernangebote eng an die Frage der Modularität gebunden. Module sind als Teil eines Ganzen definiert [vgl. Sloa02]. Hinsichtlich der Kriterien, die die Granularität von Lernangeboten bestimmen, kommt man allerdings in der technischen und in der pädagogischen Literatur zu unterschiedlichen Ergebnissen. Aus technischer Perspektive wird die Modularität bzw. Granularität von Lerneinheiten bereits länger diskutiert [Eck $\left.{ }^{+} 02\right]$. Ziele der Modularisierung sind u.a. [Balz92, S. 573]:

- Vereinfachung der Arbeitsorganisation und -planung;

- hohe Änderungsfreundlichkeit,

- Erleichterung der Standardisierung und

- Realisierung des Austausches.

Spezifische Fragestellungen beschäftigen sich mit den Problemen, wie diese technischen Module verwaltet werden können, welche technischen Kriterien und 
Standards gelten müssen, damit sie (re-)kombinierbar werden und wie die Ersteller von Modulen (Autoren) unterstützt werden. Die Module - so die Vorstellung können dadurch von verschiedenen Autoren entwickelt werden, sie greifen dennoch inhaltlich ineinander und machen zusammen eine Lerneinheit aus [ $\left.\operatorname{Eck}^{+} 02\right]$.

Ein solchermaßen technisch akzentuiertes Modul besteht also aus verschiedenen inhaltlich zusammenhängenden Multimedia-Objekten, die zusammengefasst eine oder mehrere Bildschirmseiten füllen. Die Größe bzw. Granularität von Modulen bestimmt sich aus dem zu Grunde liegenden fachwissenschaftlichen Inhalt bzw. dessen Struktur und Aufbau [vgl. z. B. FiRo02]. Das Granularitätskriterium setzt sich nach Balzert aus zwei gegenläufigen Relationen zusammen: die inhaltliche Kupplung zwischen den Modulen muss möglichst gering sein, während die inhaltliche Kohärenz innerhalb eines Moduls möglichst hoch sein muss [Balz92]. Diese Annahme kann durchaus dazu führen, dass auf gleicher Ebene Lernsequenzen mit stark unterschiedlichen Größen entstehen.

Unserer Meinung nach bestimmt sich die Größe bzw. Granularität von Lernsequenzen jedoch entgegen der technischen Vorstellung nicht ausschließlich über den fachlich zu Grunde liegenden Inhalt. Aus pädagogischer Perspektive müssen zunächst lernpsychologische Kriterien bei der Granularität der Lerneinheiten einbezogen werden. Bei diesen Kriterien handelt es sich um Fragestellungen, die die Aufmerksamkeitssteuerung bzw. die kognitiven Verarbeitungsmöglichkeiten der Lernenden betreffen. Der Umfang einer Lernsequenz umfasst aus lerntheoretischer Perspektive daher nicht nur die Bildschirmgröße, sondern auch den benötigten Zeitumfang zum Lesen bzw. Bearbeiten. Neben diesen lernpsychologischen Kriterien gilt es pädagogische Intention, methodische Umsetzung sowie mediale Aufbereitung zu berücksichtigen.

Die methodische und mediale Aufarbeitung eines fachlichen Inhalts zu Lerninhalt mit einer pädagogischen Intention wird als Prozess der didaktischen Transformation [vgl. Möhl82] bzw. Modellierung [vgl. Ach ${ }^{+}$92; Acht01] betrachtet. Sowohl dem Erstellen von Modulen als auch dem erneuten Zusammenstellen zu Lernsequenzen liegen Prozesse der didaktischen Modellierung zu Grunde. Eine didaktische Kohärenz ist daher mit der von Balzert formulierten inhaltlichen Kohärenz nicht vorbehaltlos gleichzusetzen.

Nur didaktisch ausgestaltete Lerninhalte mit aufeinander abgestimmten und an der jeweiligen didaktischen Funktion orientierten Komponenten können in unterschiedlichen Lernsituationen eingesetzt werden. Benötigt werden daher zunächst didaktisch begründete Vorgaben für die Autoren zur Entwicklung lernergerechter Inhalte. Diese Vorgaben sind in Document Type Definitions zu verankern. Darauf ausgerichtete Lerninhalte können dann ergänzend mit Metadaten semantisch angereichert werden, um im Rahmen des redaktionellen Content Management (im engeren Sinne [vgl. Ger $\left.\left.{ }^{+} 02 \mathrm{a}\right]\right)$ in alternativen Kontexten wiederverwendet zu werden. 


\subsection{Didaktische Akzentuierung der Strukturdefinitionen}

Soll eine Strukturierung und Sequenzierung des Lernangebots über eine DTD erfolgen, so müssen unserer Meinung nach die didaktisch-methodischen Komponenten ebenso wie deren Umsetzung in curriculare Einheiten und deren Ausgestaltung in mediale Präsentationsformen als Elemente innerhalb der DTD festgelegt werden. Die Abgrenzung einzelner Elemente innerhalb einer DTD bestimmt sich aus diesem Grund nicht allein über den unterschiedlichen Aufbau der einzelnen Elemente, sondern vor allem über ihre Funktion bzw. Stellung innerhalb des LehrLern-Prozesses.

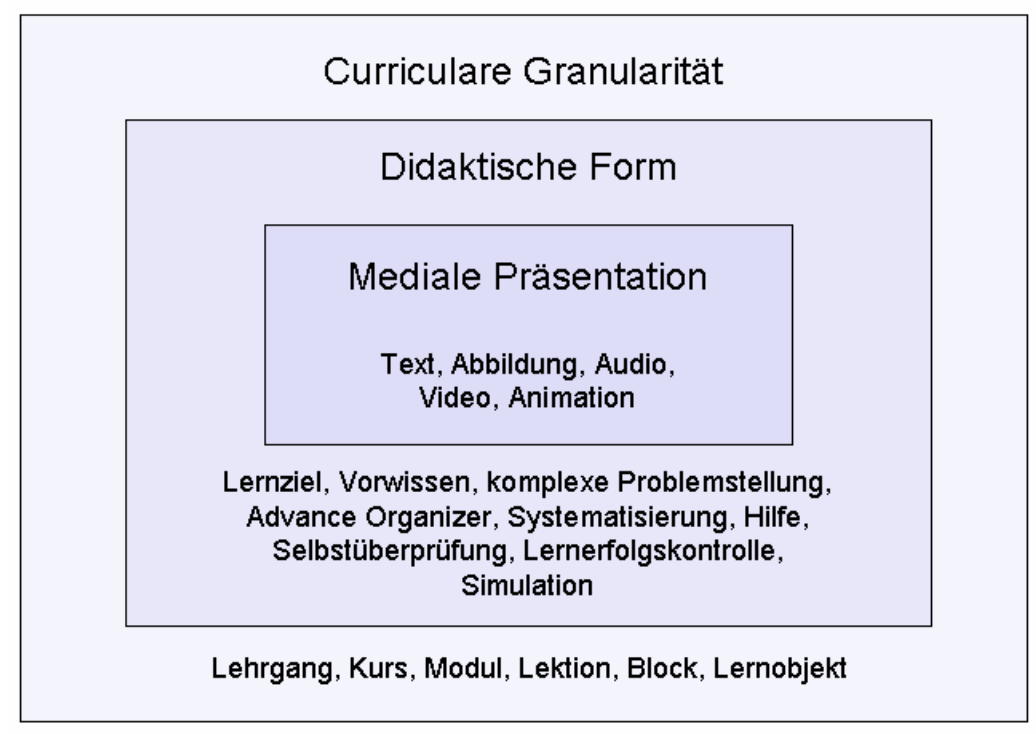

Abbildung 3: Arten von DTD-Elementtypen für Lerninhalte

Die Autoren entwickelten im Projekt $\operatorname{IMPULS}^{\mathrm{EC}}$ in interdisziplinärer Zusammenarbeit die $P B L$-DTD für die strukturierte Aufbereitung von Lerninhalten nach Gestaltungsprinzipien des Problem-Based Learning zum Thema E-Commerce.

Innerhalb dieser DTD werden die folgenden Arten von Elementtypen unterschieden (vgl. Abbildung 3):

1. Elementtypen mit Präsentationsfunktion legen die mediale Aufbereitungsart der Lerninhalte fest.

2. Elementtypen mit mikrosequenzieller Funktion setzen die didaktischmethodischen Komponenten innerhalb der DTD um. Sie besitzen eine didaktische Funktion für den Lernprozess und werden medial präsentiert. 
3. Elementtypen mit makrosequenzieller Funktion klären die Frage der curricularen Granularität und Modularität. In einem sinnvoll aufbereiteten Lernangebot bestehen sie aus didaktisch aufbereiteten Lerninhalten.

Detaillierte Ausführungen zu den einzelnen DTD-Elementen mit ihrer didaktischer Funktion sind unseren Arbeitsberichten zu entnehmen $\left[\mathrm{Ger}^{+} 02 \mathrm{~b} ; \mathrm{Kla}^{+} 02\right.$; $\left.\mathrm{Jun}^{+} 02 ; \mathrm{Jun}^{+} 03\right]$.

Eine spätere Wiederverwendung der Elemente ist von der Rolle der Person abhängig, die die Lerninhalte zusammenstellt. Autoren, Tutoren und Lernende verfügen diesbezüglich über unterschiedliche Qualifikationen. DTD-Elemente mit medialer Präsentation können einzeln wiederverwendet werden, sind dann jedoch nicht als didaktische Einheit zu betrachten. Diese Möglichkeit muss gegeben sein, damit kostenintensive multimediale Komponenten mehrfach verwendet werden können. Eine Wiederverwendung ist in diesem Fall jedoch nur dann möglich, wenn die entsprechenden Elemente von didaktisch ausgebildeten Autoren in eine didaktische Struktur wie z. B. der $P B L$-DTD eingearbeitet werden. Im Gegensatz dazu sind von Tutoren nur Elemente mit curricularer Granularität, d. h. didaktisch aufbereitete Lerninhalte, sinnvoll wiederverwendbar.

\subsection{Konsequenzen für die Wiederverwendung von Lerninhalten}

Der bislang diskutierte Lösungsansatz der PBL-DTD ist geprägt durch eine darin vollzogene weitgehende Integration von didaktischen und informationstechnischen Konzepten. Im Projekt IMPULS ${ }^{\mathrm{EC}}$ wurde die Vorteilhaftigkeit des Ansatzes für die informationstechnische Kontrolle didaktisch geforderter Regelungen und Strukturen bereits exemplarisch nachgewiesen. Im Projekt entstehen didaktisch, strukturell und medial konsistente, modulare Lerninhalte in Form von Kursen, Modulen, Lektionen, Blöcken und Lernobjekten mit deutlich höherem Ähnlichkeitsgrad auf jeder Hierarchiestufe, als es ohne die semantische Modellierung der Fall sein könnte.

Für die Unterstützung des aus ökonomischer Sicht erstrebenswerten Konzeptes der Wiederverwendung genügt die $P B L$-DTD allein jedoch noch nicht. Es existiert Bedarf an zusätzlicher semantischer Modellierung. Nur dann ist eine systemseitige Unterstützung der Klassifikation, Identifikation, Selektion und Rekombination von Lerninhalten (auf unterschiedlichen Hierarchiestufen) im Rahmen der Wiederverwendung in neuem Kontext möglich. Neben der Frage der fachinhaltlichen "Passfähigkeit", die grundsätzlich durch Metadaten in Form von Schlüsselwörtern, Thesauri und Dokumentvektoren im Rahmen des Information Retrieval gelöst werden kann, sind jedoch vorrangig die folgenden Aspekte der didaktischen Makrosequenzierung zu berücksichtigen (vgl. dazu die in Kapitel 1 aufgeführten Formen der Wiederverwendung): 
- Zielgruppen mit Vorwissen, Interessen und Motivation,

- Lernpfade mit didaktisch festgelegten Vorgänger-/Nachfolger-Relationen,

- Lernziele und

- weitere externe Vorgaben (z. B. Zeit, Infrastruktur, Medien, Rolle der Nutzer).

Deshalb ist es notwendig, mehrdimensionale Metadaten-Strukturen zu definieren, wie sie im Rahmen der Produkttaxonomie auf elektronischen Märkten bekannt sind. Dort wird die Beschreibung hierarchischer, logisch verknüpfter Produktgruppen zu Produktgruppenbäumen um Merkmalsausprägungen auf Basis standardisierter Attributmengen ("Sachmerkmalsleisten") ergänzt [OtBe01]. Für diese Detaillierung unserer PBL-DTD sind die Spezifikationen (z. B. LOM) nicht ausreichend.

Wie wir festgestellt haben, ergibt sich jedoch im praktischen Einsatz der Vergabe von Metadaten bei der Entwicklung wiederverwendbarer Lerninhalte das Problem der "Überspezifikation". In Folge der damit verbundenen Überforderung der - didaktisch, nicht technisch geprägten - Autoren kommt es schnell zu mangelnder Akzeptanz der Methode und der Werkzeuge, mithin zu kontraproduktiven Reaktionen. Ein Ausbalancieren der unterschiedlichen Interessen aus Sicht informationstechnischer und didaktischer Modellierung einerseits sowie didaktischer und fachinhaltlicher Umsetzung andererseits ist notwendig. Hier sehen wir in Ergänzung zur didaktischen Strukturierung weiteren Handlungsbedarf, den wir im Projektfortschritt auf der Ebene des Content Managements, ergänzt um die externe semantische Modellierung in Form von XML Topic Maps, untersuchen [WiMü02; PeMo02].

\section{Fazit und Ausblick: Qualität durch Interdisziplinarität}

XML-basierter Content für E-Learning-Angebote wird derzeit primär aus informationstechnischer Perspektive betrachtet. Während bei anderen XML-Anwendungskontexten in der Regel eine Verwendung von Dokumenten durch den Nutzer erzielt wird, besteht die Aufgabe in diesem Anwendungsfeld hingegen darin, den Lernenden konsistente Lerninhalte zur Verfügung zu stellen, die die kognitive Aneignung von Lerninhalten und die metakognitive Reflexion des Aneignungsprozesses durch den Lernenden unterstützen. Insofern bezieht sich eine Bearbeitung des Inhalts im XML-Anwendungsgebiet E-Learning nicht auf eine organisatorische (Weiter-)Verarbeitung der Dokumente, sondern zielt auf die kognitive Nutzung durch den Lernenden. Dokumente eines E-Learning-Angebots sind daher entsprechend ihrer didaktischen Intention zu modellieren und sollten nur innerhalb eines didaktischen Zusammenhangs wiederverwendet werden. Der Einsatz von 
XML im Bereich E-Learning bezieht die Frage der Wiederverwendung auf den Lerninhalt, nicht auf den Content. Die semantischen Modelle müssen den beabsichtigten Lernprozess, die didaktische Funktion der jeweiligen Lernsequenzen und ihre methodische und multimediale Gestaltung gleichermaßen berücksichtigen.

Die technisch geprägte Vorstellung, dass aus der Verbindung von zwei „neutralen“ Lernobjekten automatisch ein „,neuer, sinnvoller“ Lerninhalt entsteht und die Rekombination sogar vollständig automatisiert werden könnte, ist zunächst nicht realistisch. Aus didaktischer Perspektive ist dieses Vorgehen, wie es derzeit von Standardisierungsinitiativen vertreten und in der (informationstechnischen) Literatur diskutiert wird, nicht ohne weiteres akzeptabel. Es genügt nicht, Inhalte in strukturgleiche Module zu unterteilen sowie mit inhaltsbezogenen Metadaten zu versehen, um sie dann unabhängig von ihrer didaktischen Funktion in verschiedenen Zusammenstellungen wiederverwenden zu können. Kennzeichnend für eine didaktisch sinnvolle Rekombination von Lerninhalten kann nicht deren technische Strukturgleichheit, sondern muss die Funktion der Module im Lernprozess sowie deren didaktische Kohärenz sein. Diese Anforderungen können in Metadaten nur ansatzweise umgesetzt werden. Unserer Meinung nach ist neben der Vergabe von Metadaten eine didaktische Strukturierung notwenig. Bereits bestehende technische Konzepte bzw. Denkweisen müssen folglich kritisch hinterfragt und gegebenenfalls unter didaktischer Perspektive modifiziert werden.

Wie der vorliegende Beitrag zeigt, ist für die Wiederverwendung von Lerninhalten ein didaktischer Rahmen erforderlich. Dieser kann in DTDs durch eine inhaltsorientierte Strukturierung vorgegeben werden. Daneben sind zusätzliche, ebenfalls vorrangig didaktisch geprägte Metadaten Voraussetzung für die Wiederverwendung. Ein interdisziplinäres Zusammenspiel von Didaktik und Informationsmanagement ist erforderlich, um die didaktische und die ökonomische Effizienz von ELearning-Angeboten bei erforderlicher hoher Qualität zu gewährleisten. Konkrete Handlungsempfehlungen können derzeit noch nicht gegeben werden - wir stehen erst am Anfang. Eine Schlussfolgerung kann allerdings bereits zu diesem Zeitpunkt gezogen werden: diese Fragestellungen werden in Zukunft verstärkt interdisziplinär diskutiert werden müssen.

\section{Literatur}

[Ach ${ }^{+}$92] Achtenhagen, F.; Tramm, T.; Preiß, P.; Seemann-Weymar, H.; John, E. G.; Schunck, A.: Lernhandeln in komplexen Situationen. Neue Konzepte der betriebswirtschaftlichen Ausbildung. Wiesbaden: Gabler, 1992.

[Acht01] Achtenhagen, F.: Criteria for the development of complex teaching-learning environments. Instructional Science, 29 (July-Sept), 2001, S. 361-380. 
[ADL03] ADL: SCORM. http://www.adlnet.org/, Abruf am 2003-01-29.

[Balz92] Balzert, H. (Hrsg.): Die Entwicklung von Software-Systemen. Prinzipien, Methoden, Sprachen, Werkzeuge. BI-Wissenschaftsverlag: Mannheim, 1992.

[Balz98] Balzert, H.: Lehrbuch der Software-Technik. Spektrum: Heidelberg, 1998.

[Diet02] Dietzsch, A.: Systematische Wiederverwendung in der Software-Entwicklung. Dissertation, Technische Universität Dresden, 2002.

[DuJo92] Duffy, T. M.; Jonassen, D. H. (Eds.): Constructivism and the technology of instruction: A conversation. Erlbaum: Hillsdale, 1992.

[Eck $\left.{ }^{+} 02\right]$ Eckelmans, F.; Haas, C.; Hoppe, U.; Packmohr, S.: The concept of modularization in the development of online learning software - an effort to clarify from a pedagogical and a software engineering view. In: Flückiger, F.; Jutz, C.; Schulz, P.; Cantoni, L. (Hrsg.). 4th International Conference on New Educational Environment. Sauerländer: Lugano, 2002, S. 19-22.

[FiRo02] Finsterle, L.; Rotard, M.: Mit konventionellen Autorensystemen zum E-Learning Portal. In: Jantke, K. P.; Wittig, W. S.; Herrmann, J. (Hrsg.). Von e-Learning bis ePayment. Das Internet als sicherer Marktplatz. Tagungsband LIT '02; 26./27. September 2002, Leipzig. Akad. Verl.-Ges. AKA: Berlin, 2002, S. 111-121.

[Fran00] Frank, U.: Vergleichende Betrachtung von Standardisierungsvorhaben zur Realisierung von Infrastrukturen für das E-Business. Arbeitsberichte des Instituts für Wirtschaftsinformatik: Koblenz-Landau, 2000.

[Ger ${ }^{+}$02a] Gersdorf, R.; Jungmann, B.; Schoop, E.: Content Management. Vergleichende Buchbesprechung. Wirtschaftsinformatik, 44 (1), 2002, S. 79-85.

[Ger $\left.{ }^{+} 02 b\right]$ Gersdorf, R.; Jungmann, B.; Schoop, E.; Wirth, K.; Klauser, F.: Chancen und Herausforderungen bei der Abbildung didaktischer Anforderungen in XML. In: Jantke, K. P.; Wittig, W. S.; Herrmann, J. (Hrsg.). Von e-Learning bis e-Payment. Das Internet als sicherer Marktplatz. Tagungsband LIT '02; 26./27. September 2002, Leipzig. Akad. Verl.-Ges. AKA: Berlin, 2002, S. 339-346.

[GoPr00] Goldfarb, C. F.; Prescod, P.: XML-Handbuch. 2., akt. und erw. Aufl., AddisonWesley: München, 2000.

[Griff98] Griffel, F.: Componentware: Konzepte und Technologien eines Softwareparadigmas. dpunkt: Heidelberg, 1998.

[Häf $\left.{ }^{+} 02\right]$ Häfele, H.; Häfele, K.; Baumgartner, P.: E-Learning: Didaktische und technische Grundlagen. bm:bwk, 2002.

[Jun ${ }^{+}$02] Jungmann, B.; Wirth, K.; Klauser, F.; Schoop, E.: IKURS: Integrative Konzeption und Umsetzung curricularer, didaktisch-methodischer und informationstechnischer Aspekte in Richtlinien und Strukturmodelle für die Ausgestaltung multimedialer LehrLern-Arrangements. In: Bogaschewsky, R., Hoppe, U., Klauser, F., Schoop, E., Weinhardt, Ch. (Hrsg.). Research Report Impuls ${ }^{\mathrm{EC}}$ 2. Osnabrück, 2002.

[Jung03] Jungmann, B.: Einsatz von XML zur Abbildung von Lerninhalten für E-LearningAngebote: Standards, Anwendung, Handlungsbedarf. In: Esswein, W.; Schoop, E.; Uhr, W. (Hrsg.). Dresdner Beiträge zur Wirtschaftinformatik, Nr. 41/03, Dresden, 2003. 
[Jun ${ }^{+}$03] Jungmann, B.; Petzoldt, O.; Wirth, K.; Klauser, F.; Schoop, E. (im Druck): Didaktische Funktionen und deren Ausgestaltung in DTDs. Interdisziplinäres Regelwerk im E-Learning. In: Bogaschewsky, R., Hoppe, U., Klauser, F., Schoop, E., Weinhardt, Ch. (Hrsg.). Research Report Impuls ${ }^{\mathrm{EC}}$ 4. Osnabrück, 2003.

[Klau98a] Klauser, F.: "Anchored Instruction" - Eine Möglichkeit zur effektiven Gestaltung der Lehr-Lern-Prozesse in der kaufmännischen Ausbildung. Erziehungswissenschaft und Beruf, 1998 (46), S. 283-305.

[Klau98b] Klauser, F.: Problem-Based Learning - ein curricularer und didaktischmethodischer Ansatz zur innovativen Gestaltung der kaufmännischen Ausbildung. Zeitschrift für Erziehungswissenschaft, 1998, 1 (2), S. 273-293.

[Klau98c] Klauser, F.: Problem-Based Learning - ein innovativer Ansatz für die kaufmännische Ausbildung. Schweizerische Zeitschrift für kaufmännisches Bildungswesen, 1998, 92 (5), S. 330-354.

[Klau02] Klauser, F.: E-Learning problembasiert gestalten. In: Hohenstein, A.; Wilbers, K. (Hrsg.). Handbuch E-Learning. Expertenwissen aus Wissenschaft und Praxis - Strategien, Instrumente, Fallstudien. 1. Ergänzungslieferung - August 2002, Deutscher Wirtschaftsdienst: Köln, 2002, Kap. 4.12, S. 1-15.

[Kla ${ }^{+}$02] Klauser, F.; Schoop, E.; Gersdorf, R.; Jungmann, B.; Wirth, K.: Die Konstruktion komplexer internetbasierter Lernumgebungen im Spannungsfeld von pädagogischer und technischer Rationalität. In: Bogaschewsky, R., Hoppe, U., Klauser, F., Schoop, E., Weinhardt, Ch. (Hrsg.). Research Report Impuls ${ }^{\mathrm{EC}}$ 3. Osnabrück, 2002.

[K1St01] Klein, M.; Stucky, W.: Ein Vorgehensmodell zur Erstellung virtueller Bildungsinhalte. Wirtschaftsinformatik, 43 (1), 2001, S. 35-45.

[Krcm00] Krcmar, H.: Informationsmanagement. Springer Verlag: Berlin, 2000.

[Lobi00] Lobin, H.: Informationsmodellierung in XML und SGML. Springer Verlag: Berlin, 2000.

[Luc ${ }^{+}$02] Lucke, U.; Wiesner, A.; Schmeck, H.: XML: Nur ein neues Schlagwort? - Zum Nutzen von XML in Lehr-und Lernsystemen. it + ti, (4), 2002, S. 211-216.

[Man ${ }^{+}$22] Mandl, H.; Gruber, H.; Renkl, A.: Situiertes Lernen in multimedialen Lernumgebungen. In: Issing, L. J.; Klimsa, P. (Hrsg.). Information und Lernen mit Multimedia und Internet. 3. Aufl., Beltz: Weinheim, 2002, S. 139-148.

[Möhl82] Möhlenbrock, R.: Modellbildung und didaktische Transformation. Bad Salzdetfurth: Franzbecker, Didaktischer Dienst, 1982.

[OtBe01] Otto, B.; Beckmann, H.: Klassifizierung und Austausch von Produktdaten auf elektronischen Marktplätzen. Wirtschaftsinformatik, 43 (4), 2001, 351-362.

[PaAd01] Pawlowski, J. M.; Adelsberger, H. H.: Standardisierung von Lerntechnologien. Wirtschaftsinformatik, 43 (1), 2001, S. 57-68.

[PeMo02] Pepper, S.; Moore, G.: XML Topic Maps (XTM) 1.0 Specification. http://www.topicmaps.org/xtm/index.html. 2002. 
[Reza95] Rezagoholi, M.: Management der Wiederverwendung in der Softwareentwicklung. Wirtschaftsinformatik, 37 (3), 1995, S. 221-230.

[RoRi01] Rothfuss, G.; Ried, C.: Content Management mit XML. Springer Verlag: Berlin, 2001.

[Schr97] Schraml, T.: Operationalisierung der ökologiebezogenen Berichterstattung aus Sicht des Informationsmanagements. Konzeption eines Vorgehensmodells zur formalisierten Explikation logischer Dokumenttypmodelle im Rahmen der Umweltkommunikation von Unternehmen. Dissertation, Technische Universität Dresden, 1997.

[Sloa02] Sloane, P. F. E.: Modularisierung in der beruflichen Ausbildung - oder: Die Suche nach dem Ganzen. In: Euler, D.; Sloane, P. F. E. (Hrsg.). Duales System im Umbruch: Eine Bestandsaufnahme der Modernisierungsdebatte. Centaurus-Verl.-Ges.: Pfaffenweiler, 2002, S. 223-245.

[Webe92] Weber, H.: Die Software-Krise und ihre Macher, Springer: Berlin, 2002.

[WiMü02] Widhalm, R.; Mück, T.: Topic Maps. Semantische Suche im Internet. Xpert.press: Berlin, 2002. 